\title{
Existence and uniqueness of weak positive solution for es- sential singular elliptic problem involving the square root of the Laplacian
}

\author{
Xing Wang ${ }^{\mathrm{a}, *}$, Li Zhang ${ }^{\mathrm{b}}$ \\ a School of Science, Xi'an University of Technology, Xi'an, Shaanxi 710054, P. R. China. \\ ${ }^{b}$ School of Science, Chang'an University, Xi'an, Shaanxi 710064, P. R. China.
}

Communicated by J. J. Wei

\begin{abstract}
In this paper we consider the existence and uniqueness of weak positive solution for nonlocal equations of the square root of the Laplacian with singular nonlinearity. The remarkable feature of this paper is the fact that the natural associated functional fails to be Fréchet differentiable, critical point theory could not be applied to obtain the existence of weak positive solution. We first establish the priori estimate of weak solution of approximating problems. Then the weak positive solution is constructed by combining sub-and supersolutions method and truncate technology.
\end{abstract}

Keywords: Fractional Laplacian, essential singular nonlinearity, nondifferentiable functional, a priori estimate.

2010 MSC: 35J75, 47J05, 35J25.

(C)2018 All rights reserved.

\section{Introduction and preliminaries}

In this paper we study the existence of weak positive solution for the following singular elliptic boundary value problem involving a nonlocal positive operator-the square root of the Laplacian in a bounded domain

$$
\begin{cases}(-\Delta)^{\frac{1}{2}} u=f(x) u^{-\gamma}, & \text { in } \Omega \\ u>0, & \text { in } \Omega \\ u=0, & \text { on } \partial \Omega\end{cases}
$$

where $\Omega \subset R^{N}(N \geqslant 2)$ is a bounded domain, $\gamma>0$ is a real parameter, $f \in L^{p}(\Omega), f \geqslant 0, f \not \equiv 0$.

Essential to the results in this work is to realize the nonlocal operator $(-\Delta)^{\frac{1}{2}}$ in a local manner. Moreover, using this approach we can obtained the weak positive solution for the singular elliptic problem with essential singular nonlinearity, i.e., $\gamma>1$.

\footnotetext{
*Corresponding author

Email address: xj19856@sina.com (Xing Wang)

doi: $10.22436 /$ jnsa.011.10.04
}

Received: 2018-04-15 Revised: 2018-06-15 Accepted: 2018-06-19 
Before stating the results contained in this work, let us recall the definition of the fractional Laplacian and the results of the related works on fractional problem with singular nonlinearity. See for instance $[9,10]$. Given a bounded smooth domain $\Omega \subseteq R^{N}$, we consider the Sobolev space

$$
\mathrm{H}_{0, \mathrm{~L}}^{1}(\mathcal{C})=\left\{v \in \mathrm{H}^{1}(\mathcal{C}) \mid v \equiv 0 \text { a.e. on } \partial_{\mathrm{L}} \mathcal{C}\right\}
$$

equipped with

$$
\|v\|=\left(\int_{\mathcal{C}}|\nabla v|^{2} \mathrm{~d} x \mathrm{~d} y\right)^{\frac{1}{2}}
$$

where $\mathcal{C}=\Omega \times(0, \infty), \partial_{\mathrm{L}} \mathcal{C}=\partial \Omega \times[0, \infty)$. For a fixed parameter $\mathrm{s} \in(0,1)$, the fractional Laplacian in $\mathrm{R}^{\mathrm{N}}$ is a pseudo differential operator defined as

$$
\begin{aligned}
(-\Delta)^{s} u(x) & :=C_{N}, s \text { P.V. } \int_{R^{N}} \frac{u(x)-u(y)}{|x-y|^{N+2 s} d y \quad x \in R^{N},} \\
& =C_{N}, s \lim _{\varepsilon \rightarrow 0^{+}} \int_{R^{N} \backslash B(x, \varepsilon)} \frac{u(x)-u(y)}{|x-y|^{N+2 s}} d y \quad x \in R^{N},
\end{aligned}
$$

where P.V. stands for the Cauchy principle value, $B(x, \varepsilon)$ is the ball centered at $x \in R^{N}$ with radius $\varepsilon$, and $C_{N, s}$ is the following positive normalization constant:

$$
\mathrm{C}_{\mathrm{N}, \mathrm{s}}=\left(\int_{\mathrm{R}^{\mathrm{N}}} \frac{1-\cos \left(\eta_{1}\right)}{|\eta|^{\mathrm{N}+2 s}} \mathrm{~d} \eta\right)^{-1}=\pi^{-\frac{n}{2}} 2^{2 s} \frac{\Gamma\left(\frac{\mathrm{n}+2 \mathrm{~s}}{2}\right)}{\Gamma(1-\mathrm{s})},
$$

with $\eta=\left(\eta_{1}, \eta_{2}\right), \eta_{2} \in R^{N-1}$.

Given the $s \in(0,1)$ and a smooth bounded domain $\Omega \subseteq \mathrm{R}^{\mathrm{N}}$, we can defined the fractional Sobolev space as

$$
H^{s}(\Omega)=\left\{u \in L^{2}(\Omega) \mid \int_{\Omega} \int_{\Omega} \frac{|u(x)-u(y)|^{2}}{|x-y|^{n+2 s}} d x d y<+\infty\right\}
$$

with norm

$$
\|u\|_{H^{s}}=\left\{\int_{\Omega}|u|^{2} d x+\int_{\Omega} \int_{\Omega} \frac{|u(x)-u(y)|^{2}}{|x-y|^{n+2 s}} d x d y\right\}^{\frac{1}{2}} .
$$

We define $H_{0}^{s}(\Omega)$ as the closure of $C_{c}^{\infty}(\Omega)$ with respect to the norm $\|\cdot\|_{H^{s}}$. Theorem 11.1 in [9] showed that for $0<s \leqslant \frac{1}{2}, H_{0}^{s}(\Omega)=H^{s}(\Omega)$, while for $\frac{1}{2}<s<1$ the inclusion $H_{0}^{s}(\Omega) \subseteq H^{s}(\Omega)$ is strict.

Caffarelli and Silvestre [4] have given a new local realization of the fractional Laplacian operator $(-\Delta)^{s}(s \in(0,1))$, through the Dirichlet-Neumann map of an appropriate degenerate elliptic operator. By a weak solution $u$ of $(1.1)$ we mean look for $v \in \mathrm{H}_{0, \mathrm{~L}}^{1}(\mathrm{C})$ with $v(\cdot, 0)=u$, satisfying the following mixed boundary value problem

$$
\begin{cases}-\Delta v=0, & \text { in } \mathcal{C}, \\ v=0, & \text { on } \partial_{\mathrm{L}} \mathcal{C}, \\ v>0, & \text { in } \mathcal{C}, \\ \frac{\partial v}{\partial \mathbf{n}}=\mathrm{f}(\mathrm{x}) v^{-\gamma}, & \text { on } \Omega \times\{0\},\end{cases}
$$

where $\mathbf{n}$ is the unit outer normal to $\mathcal{C}$ at $\Omega \times\{0\}$.

Definition 1.1. We say $v \in \mathrm{H}_{0, \mathrm{~L}}^{1}(\mathcal{C})$ is a weak supersolution(respectively subsolution) of equation (1.2), if $v \geqslant 0$ and

$$
\int_{\mathcal{C}} \nabla v \nabla \phi \geqslant(\leqslant) 0, \quad \forall \phi \in \mathrm{H}_{0, \mathrm{~L}}^{1}(\mathcal{C})
$$

and

$$
\int_{\mathcal{C}} \frac{\partial v}{\partial \mathbf{n}} \phi \mathrm{d} x \mathrm{~d} y \geqslant(\leqslant) \int_{\Omega \times\{0\}} f(x) v^{-\gamma} \phi \mathrm{d} x, \quad \forall \phi \in \mathrm{H}_{0, \mathrm{~L}}^{1}(\mathcal{C}) .
$$


Definition 1.2. We say $u$ is a weak supersolution(respectively subsolution) of equation (1.1), if there exists a weak supersolution(respectively subsolution) $v \in \mathrm{H}_{0, \mathrm{~L}}^{1}(\mathcal{C})$ of $(1.2)$, such that $u=\left.\operatorname{tr}\right|_{\Omega} v$.

Definition 1.3. We say $v$ is a weak positive solution of equation (1.2), if it is at the same time a supersolution and a subsolution of equation (1.2). If $v$ is a weak positive solution of equation (1.2), then the trace $u$ on $\Omega$ of $v$ is said to be a weak positive solution of (1.1).

The principal feature of interest here is that the given boundary value makes the equation singular at the boundary $\partial \Omega$. Therefore, for which class of the testing function $\phi$ makes (1.2) hold true is essential. It is worth pointing out that the testing function $\phi$ in the definition of weak positive solution dose not need to has compact support in $\Omega$. There are the following important differences between the singular elliptic equations and the general elliptic equations. That is the validity of the relation

$$
\int_{\mathcal{C}} \frac{\partial v}{\partial \mathbf{n}} \phi \mathrm{d} x \mathrm{~d} y=\int_{\Omega \times\{0\}} f(x) v^{-\gamma} \phi \mathrm{d} x, \quad \forall \phi \in \mathrm{H}_{0, \mathrm{~L}}^{1}(\mathcal{C})
$$

cannot imply the validity of

$$
\int_{\mathcal{C}} \frac{\partial v}{\partial \mathbf{n}} \varphi \mathrm{d} x \mathrm{~d} y=\int_{\Omega \times\{0\}} f(x) v^{-\gamma} \varphi \mathrm{d} x, \quad \forall \varphi \in \mathrm{C}_{\mathrm{c}}^{\infty}(\mathcal{C})
$$

since, if $\left\{\varphi_{\mathrm{n}}\right\} \subset \mathrm{C}_{\mathrm{c}}^{\infty}(\mathcal{C})$ such that $\varphi_{\mathrm{n}} \longrightarrow \varphi$ in $\mathrm{H}_{0, \mathrm{~L}}^{1}(\mathcal{C})$ topology, it is not true in general that

$$
\int_{\Omega \times\{0\}} f(x) u^{-\gamma} \varphi_{n} d x \longrightarrow \int_{\Omega \times\{0\}} f(x) u^{-\gamma} \varphi d x
$$

Problem (1.1) is strongly inspired by the following semilinear elliptic problem with a singular nonlinearity

$$
\begin{cases}-\Delta u=\lambda u^{\beta}+p(x) u^{-\gamma}, & \text { in } \Omega, \\ u=0, & \text { on } \partial \Omega .\end{cases}
$$

Problem (1.4) has been extensively studied by various authors (see, e.g. $[14,15,17,20]$ and the references there in). In pioneering papers of Crandall et al. [5] and Stuart [13] proved that problem (1.4) has a unique classical positive solution $u \in C^{2}(\Omega) \cap C^{0}(\bar{\Omega})$, using the method of sub-and super-solutions when $\lambda=0$, $p \in \mathrm{C}^{1}(\bar{\Omega})$. By the Ekeland's variational principle [12] and careful estimates, Sun [16] and my previous work [18] showed that the problem (1.4) possesses at least two weak solutions, provided $1<\beta \leqslant \frac{N+2}{N-2}$, $0<\gamma<1$ and $\lambda>0$ small enough.

The fractional Laplacian is the infinitesimal generator of Lévy stable diffusion process and in anomalous diffusion in plasma, population, dynamics, geophysical fluid dynamics, flames propagation, chemical reactions in liquids and American options in finance. For more details, we cite the reader to $[1,7,11]$. Problems of the type (1.1) arise in certain problems in fluid mechanics, pseudoplastic flow, chemical heterogenous catalysts and non-Newtonian fluids. The topic of the existence of weak solutions to problem (1.1) has been studied extensively in $[2,6,8,11]$ and the references there in. In [11], authors proved the existence and multiplicity results on problem (1.4), where $0<\gamma \leqslant 1$. It should be pointed out that since $1-\gamma<1$ the natural associated functional

$$
\mathrm{I}(v)=\frac{1}{2} \int_{\mathcal{C}}|\nabla v(x, y)|^{2} \mathrm{~d} x \mathrm{~d} y-\frac{1}{1-\gamma} \int_{\Omega} f(x)|v(x, 0)|^{1-\gamma} \mathrm{d} x \text { for all } v \in \mathrm{H}_{0, \mathrm{~L}}^{1}(\mathcal{C})
$$

fails to be Fréchet-differentiable. Moreover, in the essential singular case of $\gamma>1$ the functional is no defined. Therefore, the general critical point theory could not be applied to obtain the weak solution directly. To the best of our knowledge, there are no works dealing with existence results with fractional Laplacian with essential singular nonlinearities. In this paper, we attempt to address the existence of weak positive solution of problem (1.1) for all $\gamma>0$.

Throughout the paper we make use of the following notations. We denote by $|\Omega|$ the Lebesgue measure of $\Omega$ and also denote by $\|\cdot\|_{L^{q}}$ the standard $L^{q}(\Omega)$-normal for $1 \leqslant q \leqslant \infty, C, C_{1}, C_{2}, \ldots$ denote (possibly different) positive constants. 


\section{Main results}

The following theorems are the main results of this paper.

Theorem 2.1. Let $\Omega$ be a bounded smooth domain in $\mathrm{R}^{\mathrm{N}}(\mathrm{N} \geqslant 2)$. Let $\gamma \in(0,1), \mathrm{f} \in \mathrm{L}^{\mathrm{q}}(\Omega)(\mathrm{q}>\mathrm{N})$ with $\mathrm{f}(\mathrm{x}) \geqslant 0, \mathrm{f} \not \equiv 0$ in $\Omega$. Then problem (1.1) has a unique weak positive solution $\mathrm{u} \in \mathrm{L}^{\infty}(\Omega)$.

Theorem 2.2. Let $\Omega$ be a bounded smooth smooth domain in $\mathrm{R}^{\mathrm{N}}(\mathrm{N} \geqslant 2)$. Let $\gamma \geqslant 1$ and $\mathrm{f} \in \mathrm{C}^{\alpha}(\bar{\Omega})(0<\alpha<1)$ with $\mathrm{f}(\mathrm{x}) \geqslant 0, \mathrm{f} \not \equiv 0$ in $\Omega$. Assume that there exists a positive function $\eta(x) \in \mathrm{C}_{0}^{1}(\bar{\Omega})$ such that $\mathrm{f \eta}^{-\gamma} \in \mathrm{L}^{2}(\Omega)$, then problem (1.1) has a unique weak positive solution $\mathrm{u} \in \mathrm{L}^{\infty}(\Omega)$.

\section{Existence and uniqueness of weak positive solution of problem (1.1)}

Lemma 3.1 (Embedding, see [3, Lemma 2.4]). Let $\Omega$ be a bounded smooth domain in $\mathrm{R}^{\mathrm{N}}(\mathrm{N} \geqslant 2), 1<\mathrm{r} \leqslant 2^{\sharp}=$ $\frac{2 \mathrm{~N}}{\mathrm{~N}-1}$. Then, we have that for all $v \in \mathrm{H}_{0, \mathrm{~L}}^{1}(\mathrm{C})$,

$$
\left(\int_{\Omega}|v(x, 0)|^{r} \mathrm{~d} x\right)^{\frac{1}{r}} \leqslant C\left(\int_{\mathcal{C}}|\nabla v(x, y)|^{2} \mathrm{~d} x \mathrm{~d} y\right)^{\frac{1}{2}}
$$

where C depends only on $N, r$, and $|\Omega|$ the measure of $\Omega$.

Lemma 3.2 (Priori estimate). Let $\Omega$ be a bounded smooth domain in $\mathrm{R}^{\mathrm{N}}(\mathrm{N} \geqslant 3), \gamma \in(0,+\infty), \mathrm{f} \in \mathrm{L}^{\mathrm{q}}(\Omega)(\mathrm{q}>$ $\mathrm{N}), \delta \geqslant 0$ is a constant, then any weak positive solution $\mathrm{u}=\left.\operatorname{tr}\right|_{\Omega} v$ of problem $(1.1)$ belongs to $\mathrm{L}^{\infty}(\Omega)$.

Proof. We follow ideas from Moser iteration.

Assume $v \in \mathrm{H}_{0, \mathrm{~L}}^{1}(\mathcal{C})$ satisfying (1.3). For any real number $\mathrm{L}>0$ and given $\mathrm{K}>0$, we defined the truncated function

$$
v_{\mathrm{L}}(\mathrm{x})= \begin{cases}v(\mathrm{x}), & \text { if } v(x) \leqslant \mathrm{L}, \\ \mathrm{L}, & \text { if } v(x)>\mathrm{L}\end{cases}
$$

$\mathrm{G}_{\mathrm{L}}=v_{\mathrm{L}}^{2(\mathrm{~T}-1)}(v-\mathrm{K})^{+}$and $\mathrm{W}_{\mathrm{L}}=v_{\mathrm{L}}^{\mathrm{T}-1} v$ where $\mathrm{T}>1$ to be determined later. Elementary computations ensure that the functions $G_{L}$ and $W_{L}$ belong to $H_{0, L}^{1}(\mathcal{C})$ for any $T>1$. Hence, we can choose $G_{L}$ as a testing function and obtain

$$
\int_{\mathcal{C}} \frac{\partial v}{\partial \mathbf{n}} \mathrm{G}_{\mathrm{L}} \mathrm{d} x \mathrm{~d} y=\int_{\Omega \times\{0\}} f(x)(v+\delta)^{-\gamma} \mathrm{G}_{\mathrm{L}} \mathrm{dx}
$$

Taking $\mathrm{D}=\{(x, y) \in \mathcal{C} \mid v(x, y) \geqslant K\}$, we estimate the left-hand side of (3.2). We obtained that

$$
\begin{aligned}
\mathrm{LHS} & =\int_{\mathcal{C}} \frac{\partial v}{\partial \mathbf{n}} \mathrm{G}_{\mathrm{L}} \mathrm{d} x \mathrm{~d} y \\
& =\int_{\mathcal{C}} \nabla v \nabla \mathrm{G}_{\mathrm{L}} \mathrm{d} x \mathrm{~d} y \\
& =\int_{\mathcal{C}} \nabla v \nabla\left(v_{\mathrm{L}}^{2(\mathrm{~T}-1)}(v-\mathrm{K})^{+}\right) \mathrm{d} x \mathrm{~d} y \\
& =\int_{\mathrm{D}} \nabla v \nabla\left(v_{\mathrm{L}}^{2(\mathrm{~T}-1)}(v-\mathrm{K})\right) \mathrm{d} x \mathrm{~d} y \\
& =(2 \mathrm{~T}-1) \int_{\mathrm{D}} v_{\mathrm{L}}^{2(\mathrm{~T}-1)-1}(v-\mathrm{K}) \nabla v \nabla v_{\mathrm{L}} \mathrm{d} x \mathrm{~d} y+\int_{\mathrm{D}} v_{\mathrm{L}}^{2(\mathrm{~T}-1)}|\nabla v|^{2} \mathrm{~d} x \mathrm{~d} y \\
& =(2 \mathrm{~T}-1) \int_{\mathrm{D} \cap\{(x, y) \in \mathcal{C} \mid v(x, y) \leqslant \mathrm{L}\}} v_{\mathrm{L}}^{2(\mathrm{~T}-1)-1}(v-\mathrm{K}) \nabla v \nabla v_{\mathrm{L}} \mathrm{d} x \mathrm{~d} y
\end{aligned}
$$




$$
\begin{aligned}
& +(2 \mathrm{~T}-1) \int_{\mathrm{D} \cap\{(x, y) \in \mathcal{C} \mid v(x, y)>\mathrm{L}\}} v_{\mathrm{L}}^{2(\mathrm{~T}-1)-1}(v-\mathrm{K}) \nabla v \nabla v_{\mathrm{L}} \mathrm{d} x \mathrm{~d} y+\int_{\mathrm{D}} v_{\mathrm{L}}^{2(\mathrm{~T}-1)}|\nabla v|^{2} \mathrm{~d} x \mathrm{~d} y \\
= & (2 \mathrm{~T}-1) \int_{\mathrm{D} \cap\{(x, y) \in \mathcal{C} \mid v(x, y) \leqslant \mathrm{L}\}} v^{2(\mathrm{~T}-1)-1}(v-\mathrm{K})|\nabla v|^{2} \mathrm{~d} x \mathrm{~d} y \\
& +(2 \mathrm{~T}-1) \int_{\mathrm{D} \cap\{(x, y) \in \mathcal{C} \mid v(x, y)>\mathrm{L}\}} 0 \mathrm{~d} x \mathrm{~d} y+\int_{\mathrm{D}} v_{\mathrm{L}}^{2(\mathrm{~T}-1)}|\nabla v|^{2} \mathrm{~d} x \mathrm{~d} y \geqslant \int_{\mathrm{D}} v_{\mathrm{L}}^{2(\mathrm{~T}-1)}|\nabla v|^{2} \mathrm{~d} x .
\end{aligned}
$$

On the other hand

$$
\begin{aligned}
\mathrm{RHS} & =\int_{\Omega \times\{0\}} \mathrm{f}(\mathrm{x})(v+\delta)^{-\gamma} \mathrm{G}_{\mathrm{L}} \mathrm{d} x \\
& =\int_{\Omega \times\{0\}} \mathrm{f}(\mathrm{x}) v_{\mathrm{L}}^{2(\mathrm{~T}-1)}(v-\mathrm{K})^{+}(v+\delta)^{-\gamma} \mathrm{d} x \\
& \leqslant \int_{\{x \in \Omega \mid v(x, 0) \geqslant \mathrm{K}\}} \mathrm{f}(\mathrm{x}) v_{\mathrm{L}}^{2(\mathrm{~T}-1)} v^{2} v^{-1-\gamma} \mathrm{d} x \\
& \leqslant \mathrm{~K}^{-1-\gamma} \int_{\{x \in \Omega \mid v(x, 0) \geqslant \mathrm{K}\}} \mathrm{f}(\mathrm{x}) \mathrm{W}_{\mathrm{L}}^{2} \mathrm{~d} x \\
& =\mathrm{K}^{-1-\gamma}\|\mathrm{f}\|_{\mathrm{Lq}}|\Omega|^{\frac{1}{\theta}}\left(\int_{\{x \in \Omega \mid v(x, 0) \geqslant K\}} \mathrm{W}_{\mathrm{L}}^{\alpha^{\sharp}} \mathrm{dx}\right)^{\frac{2}{\alpha^{\sharp}},}
\end{aligned}
$$

where in the last step we have used the following result. There exist real parameters $\alpha^{\sharp} \in\left(2,2^{\sharp}=\frac{2 \mathrm{~N}}{\mathrm{~N}-1}\right)$ and $\theta \in(0,1)$ such that

$$
\frac{1}{\mathrm{q}}+\frac{2}{\alpha^{\sharp}}+\frac{1}{\theta}=1 \text {. }
$$

Indeed, since $\mathrm{q}>\mathrm{N}$, from simple computations, we have

$$
\frac{2}{1-\frac{1}{q}}<2^{\sharp} \text {. }
$$

Hence, we can easily choose a $\alpha^{\sharp} \in\left(\frac{2}{1-\frac{1}{q}}, 2^{\sharp}\right)$ satisfying $\frac{1}{q}+\frac{2}{\alpha^{\sharp}}<1$. Consequently, we have a $\theta>1$ such that $\frac{1}{\mathrm{q}}+\frac{2}{\alpha^{\sharp}}+\frac{1}{\theta}=1$.

Moreover, recalling the definition of $W_{\mathrm{L}}$ and using the basic formula, we obtain

$$
\begin{aligned}
\int_{\mathrm{D}}\left|\nabla \mathrm{W}_{\mathrm{L}}\right|^{2} \mathrm{~d} x \mathrm{~d} y & =\int_{\mathrm{D}}\left|\nabla\left(v_{\mathrm{L}}^{\mathrm{T}-1} v\right)\right|^{2} \mathrm{~d} x \mathrm{~d} y \\
& =\int_{\mathrm{D}}\left|(\mathrm{T}-1) v_{\mathrm{L}}^{\mathrm{T}-2} v \nabla v_{\mathrm{L}}+v_{\mathrm{L}}^{\mathrm{T}-1} \nabla v\right|^{2} \mathrm{~d} x \mathrm{~d} y \\
& \leqslant \int_{\mathrm{D}}\left|(\mathrm{T}-1) v_{\mathrm{L}}^{\mathrm{T}-1} \nabla v+v_{\mathrm{L}}^{\mathrm{T}-1} \nabla v\right|^{2} \mathrm{~d} x \mathrm{~d} y=\mathrm{T}^{2} \int_{\mathrm{D}} v_{\mathrm{L}}^{2(\mathrm{~T}-1)}|\nabla v|^{2} \mathrm{~d} x \mathrm{~d} y
\end{aligned}
$$

We deduce from (3.2), (3.3), (3.4), and (3.5) that

$$
\int_{\mathrm{D}}\left|\nabla \mathrm{W}_{\mathrm{L}}\right|^{2} \mathrm{~d} x \mathrm{~d} y \leqslant \mathrm{~T}^{2} \mathrm{~K}^{-1-\gamma}\|\mathrm{f}\|_{\mathrm{q}}|\Omega|^{\frac{1}{\theta}}\left(\int_{\{x \in \Omega \mid v(x, 0) \geqslant \mathrm{K}\}} \mathrm{W}_{\mathrm{L}}^{\alpha^{\sharp}} \mathrm{d} x\right)^{\frac{2}{\alpha^{\sharp}}} .
$$

Using the Sobolev embedding (3.1), we have

$$
\left(\int_{\Omega} \mathrm{W}_{\mathrm{L}}^{2^{\sharp}} \mathrm{d} x\right)^{\frac{2}{2 \sharp}} \leqslant \mathrm{C} \int_{\mathrm{C}}\left|\nabla \mathrm{W}_{\mathrm{L}}\right|^{2} \mathrm{~d} x \mathrm{~d} \mathrm{y}=\mathrm{C} \int_{\mathrm{D}}\left|\nabla \mathrm{W}_{\mathrm{L}}\right|^{2} \mathrm{~d} x \mathrm{~d} y
$$




$$
\leqslant C T^{2} K^{-1-\gamma}\|\mathbf{f}\|_{q}|\Omega|^{\frac{1}{\theta}}\left(\int_{\{x \in \Omega \mid v(x, 0) \geqslant K\}} W_{L}^{\alpha^{\sharp}} d x\right)^{\frac{2}{\alpha^{\sharp}}},
$$

that is

$$
\left(\int_{\mathcal{H}} u_{\mathrm{L}}^{2^{\sharp}(T-1)} \mathrm{u}^{2^{\sharp}} \mathrm{d} x\right)^{\frac{2}{2^{\sharp}}} \leqslant \mathrm{C}_{1} \mathrm{~T}^{2}\left(\int_{\mathcal{H}} \mathrm{u}_{\mathrm{L}}^{\alpha^{\sharp}(\mathrm{T}-1)} \mathrm{u}^{\alpha^{\sharp}} \mathrm{dx}\right)^{\frac{2}{\alpha^{\sharp}}},
$$

where $\mathcal{H}=\{x \in \Omega \mid u(x)=v(x, 0) \geqslant K\}, C_{1}=C T^{2} K^{-1-\gamma}\|f\|_{q}|\Omega|^{\frac{1}{\theta}}$.

From Fatou's Lemma, taking the limit as $\mathrm{L} \rightarrow+\infty$, one has

$$
\left(\int_{\mathcal{H}} u^{2^{\sharp} T} d x\right)^{\frac{2}{2^{\sharp}}} \leqslant C_{1} T^{2}\left(\int_{\mathcal{H}} u^{\alpha^{\sharp} T} d x\right)^{\frac{2}{\alpha^{\sharp}}},
$$

it follows that

$$
\|v\|_{\mathrm{L}^{\sharp} \mathrm{T}(\mathcal{H})} \leqslant \mathrm{C}_{1}^{\frac{1}{2 \mathrm{~T}}} \mathrm{~T}^{\frac{1}{\mathrm{~T}}}\|v\|_{\mathrm{L}^{\sharp} \mathrm{T}(\mathcal{H})^{\prime}}
$$

which implies that $u \in \mathrm{L}^{2^{\sharp}} \mathrm{T}(\mathcal{H})$. Letting $\mathrm{T}=\frac{2^{\sharp}}{\alpha^{\sharp}}>1$, we have $2^{\sharp} \mathrm{T}=\alpha^{\sharp} \mathrm{T}^{2}$ and consequently $\mathrm{u}^{\mathrm{T}^{2}} \in \mathrm{L}^{\alpha^{\sharp}}(\mathcal{H})$. Choosing $T^{2}$ instead of $T$ into (3.6), we infer that

$$
\begin{aligned}
\|u\|_{L^{2 \sharp} T^{2}(\mathcal{H})} \leqslant C_{1}^{\frac{1}{2 T^{2}}}\left(T^{2}\right) \frac{1}{T^{2}}\|u\|_{L^{\alpha \sharp T^{2}}(\mathcal{H})}=C_{1}^{\frac{1}{2 T^{2}}} \frac{2}{T^{2}}\|u\|_{L^{2 \sharp}(\mathcal{H})} & \leqslant C_{1}^{\frac{1}{2 T^{2}}} T^{\frac{2}{T^{2}}} C_{1}^{\frac{1}{2 T}} T^{\frac{1}{T}}\|u\|_{L^{\alpha \sharp T}(\mathcal{H})} \\
& =C_{1}^{\frac{1}{2 T^{2}}+\frac{1}{2 T}} T^{\frac{1}{T}}+\frac{2}{T^{2}}\|u\|_{L^{\alpha^{\sharp}}(\mathcal{H})} .
\end{aligned}
$$

By iterating this process, for any $m \in N$, we get

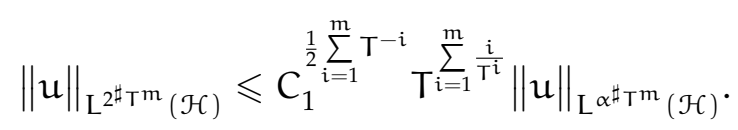

Since $T>1$ the series $\sum_{i=1}^{\infty} T^{-i}$ and $\sum_{i=1}^{\infty} \frac{i}{T^{i}}$ are convergent, passing to the limit as $m \rightarrow+\infty$, we infer that $u \in \mathrm{L}^{\infty}(\mathcal{H})$. Using the definition of set $\mathcal{H}$, the desired estimate then follows. This completes the proof of Lemma 3.2.

Proof of Theorem 2.1.

Existence. Since $0<\gamma<1$, the functional

$$
\mathrm{I}(v)=\frac{1}{2} \int_{\mathcal{C}}|\nabla v(x, y)|^{2} \mathrm{~d} x \mathrm{~d} y-\frac{1}{1-\gamma} \int_{\Omega} f(x)|v(x, 0)|^{1-\gamma} \mathrm{d} x \text { for all } v \in \mathrm{H}_{0, \mathrm{~L}}^{1}(\mathcal{C})
$$

is well defined. The difficult in this problem is that the singular term leads to the the functional $\mathrm{I}(v)$ is not Fréchet differentiable in $\mathrm{H}_{0, \mathrm{~L}}^{1}(\mathcal{C})$. Therefore, it cannot considered by using critical points theory directly. Inspired by $[16,18]$, we carry out a direct analysis in an $\mathrm{H}_{0, \mathrm{~L}}^{1}$-neighborhood and get a unique minimizer which is a weak positive solution of problem (1.2). Moreover, since $I(|v|)=I(v)$, we may assume that $v \geqslant 0$ in $\mathrm{C}$.

By using the Hölder inequality and Lemma 3.1 we infer that there exists constant $C>0$ such that

$$
\int_{\Omega} f(x)|v(x, 0)|^{1-\gamma} d x \leqslant\|f\|_{L^{q}(\Omega)}|\Omega|^{\frac{1}{\theta}}\|v(x, 0)\|_{L^{\alpha^{\sharp}(\Omega)}}^{1-\gamma} \leqslant C\|f\|_{L^{q}(\Omega)}|\Omega|^{\frac{1}{\theta}}\|v\|^{1-\gamma} .
$$

Hence, we have

$$
\mathrm{I}(v) \geqslant \frac{1}{2}\|v\|^{2}-\mathrm{C}_{1}\|v\|^{1-\gamma} \text { for all } v \in \mathrm{H}_{0, \mathrm{~L}}^{1}(\mathcal{C}) .
$$


Noting that

$$
t^{2}=o\left(t^{1-\gamma}\right)(t \rightarrow 0)
$$

there exist $\rho>0, \omega>0$ such that

$$
\mathrm{I}(v) \geqslant \frac{1}{2}\|v\|^{2}-\mathrm{C}_{1}\|v\|^{1-\gamma} \geqslant \omega \text { for all } v \in \mathrm{B}_{\rho},
$$

where $\mathrm{B}_{\rho}=\left\{v \in \mathrm{H}_{0, \mathrm{~L}}^{1}(\mathcal{C}) \mid\|v\| \leqslant \rho\right\}$. Moreover, since $0<1-\gamma<1$, we deduce that for all $v>0, \mathrm{I}(\mathrm{t} v)<0$ provided $t>0$ is sufficiently small. This implies

$$
\mathrm{m}=\inf _{v \in \mathrm{B}_{\rho}} \mathrm{I}(v)<0 .
$$

By the Theorem 1.2 in [12], we claim that there exist a $v \in \mathrm{B}_{\rho}$ such that $\mathrm{I}(v)=\mathrm{m}$. For the convenience of the reader, we sketch the main steps here.

Let $\left\{v_{n}\right\} \subseteq \mathrm{B}_{\rho}$ be a minimizing sequence such that $\mathrm{I}\left(v_{n}\right) \rightarrow \mathrm{m}$ as $\mathrm{n} \rightarrow \infty$. Using $\mathrm{H}_{0, \mathrm{~L}}^{1}(\mathcal{C})$ is a Hilbert space, we obtained a subsequence of $\left\{v_{n}\right\}$ (still denoted by $\left.\left\{v_{n}\right\}\right)$ such that $v_{n} \rightarrow v$ weakly in $\mathrm{H}_{0, \mathrm{~L}}^{1}(\mathcal{C})$ and strongly in $L^{r}(\Omega)\left(1 \leqslant r<\frac{2 N}{N-1}\right)$, and pointwise a.e. in $\Omega$. Using Hölder's inequality and (3.7), we derive that

$$
\int_{\Omega} f(x)\left|v_{n}(x, 0)\right|^{1-\gamma} d x=\int_{\Omega} f(x)|v(x, 0)|^{1-\gamma} d x+o(1) .
$$

Combining above arguments, we can get

$$
\mathrm{m}=\mathrm{I}\left(v_{\mathrm{n}}\right)+\mathrm{o}(1)=\mathrm{I}(v)+\mathrm{o}(1) \geqslant \mathrm{m}+\mathrm{o}(1)(\mathrm{n} \rightarrow \infty),
$$

that is $0 \geqslant I(v)-m+o(1) \geqslant o(1)$. Passing to the limit as $n \rightarrow \infty$, we obtain

$$
\mathrm{I}(v)=\mathrm{m} .
$$

Now, one can prove that $v$ is a weak solution of (1.3). For $\phi \in \mathrm{H}_{0, \mathrm{~L}}^{1}(\mathcal{C}), \phi \geqslant 0$ and $\varepsilon>0$, we infer

$$
\liminf _{\varepsilon \rightarrow 0^{+}} \frac{I(v+\varepsilon \phi)-I(v)}{\varepsilon} \geqslant 0,
$$

i.e.,

$$
\liminf _{\varepsilon \rightarrow 0^{+}} \frac{1}{\varepsilon}\left\{\frac{1}{2}\left(\|v+\varepsilon \phi\|^{2}-\|v\|^{2}\right)-\frac{1}{1-\gamma} \int_{\Omega} f(x)\left(|v(x, 0)+\varepsilon \phi(x, 0)|^{1-\gamma}-|v(x, 0)|^{1-\gamma}\right) d x\right\} \geqslant 0 .
$$

From simple arguments and Fatou's Lemma, we get

$$
\liminf _{\varepsilon \rightarrow 0^{+}} \frac{1}{1-\gamma} \int_{\Omega} f(x) \frac{|v(x, 0)+\varepsilon \phi(x, 0)|^{1-\gamma}-|v(x, 0)|^{1-\gamma}}{\varepsilon} d x \geqslant \int_{\Omega} f(x) v(x, 0)^{-\gamma} \phi(x, 0) d x .
$$

Moreover, noting that the functional $\|v\|$ is continuously differentiable in $\mathrm{H}_{0, \mathrm{~L}}^{1}(\mathcal{C})$, we obtained

$$
\liminf _{\varepsilon \rightarrow 0^{+}} \frac{1}{2} \frac{\|v+\varepsilon \phi\|^{2}-\|v\|^{2}}{\varepsilon}=\lim _{\varepsilon \rightarrow 0^{+}} \frac{1}{2} \frac{\|v+\varepsilon \phi\|^{2}-\|v\|^{2}}{\varepsilon}=\int_{\mathcal{e}} \frac{\partial v}{\partial \mathbf{n}} \phi d x d y .
$$

Putting together these relations we find

$$
\int_{\mathcal{C}} \frac{\partial v}{\partial \mathbf{n}} \phi d x d y-\int_{\Omega \times\{0\}} f(x) v^{-\gamma} \phi d x \geqslant 0, \quad \forall \phi \in \mathrm{H}_{0, \mathrm{~L}}^{1}(\mathcal{C}), \phi \geqslant 0 .
$$


For any $\phi \in \mathrm{H}_{0, \mathrm{~L}}^{1}(\mathcal{C})$ and $\varepsilon>0$, taking $\Phi=(v+\varepsilon \phi)^{+}$in (3.8), dividing it by $\varepsilon$ and passing to the limit as $t \rightarrow 0$, following the same arguments as in proving (3.8) we obtain

$$
\int_{\mathcal{C}} \frac{\partial v}{\partial \mathbf{n}} \phi d x d y-\int_{\Omega \times\{0\}} f(x) v^{-\gamma} \phi d x \geqslant 0, \quad \forall \phi \in \mathrm{H}_{0, \mathrm{~L}}^{1}(\mathcal{C}) .
$$

Noting that $\phi$ is arbitrary, replacing $\phi$ by $-\phi$ in (3.9), one gets the conclusion

$$
\int_{\mathcal{C}} \frac{\partial v}{\partial \mathbf{n}} \phi d x d y-\int_{\Omega \times\{0\}} f(x) v^{-\gamma} \phi d x=0, \quad \forall \phi \in \mathrm{H}_{0, \mathrm{~L}}^{1}(\mathcal{C}) .
$$

Uniqueness. Suppose by contradiction that $w \in \mathrm{H}_{0, \mathrm{~L}}^{1}(\mathcal{C})$ is also another weak positive solution of equation (1.2). Since $\left(v^{-\gamma}-w^{-\gamma}\right)(v-w) \leqslant 0$, taking $\phi=v-w$ in (3.10) one has

$$
\int_{\mathcal{C}} \frac{\partial(v-w)}{\partial \mathbf{n}}(v-w) d x d y=\int_{\Omega \times\{0\}} f(x)\left(v^{-\gamma}-w^{-\gamma}\right)(v-w) d x \leqslant 0
$$

We thus have

$$
\int_{\Omega}|\nabla(v-w)|^{2} \mathrm{~d} x \leqslant 0 \text { in } \mathcal{C} .
$$

Therefore $v-w=0$ a.e. in $\mathcal{C}$, which yields a contraction. So the uniqueness is obtained.

Moreover, with the help of Lemma 3.2, choosing $\delta=0$ we infer that $v \in \mathrm{L}^{\infty}(\mathcal{C})$. Thus $u=\left.\operatorname{tr}\right|_{\Omega} v \in$ $\mathrm{L}^{\infty}(\Omega)$.

This completes the proof of Theorem 2.1.

Next, we will prove the Theorem 2.2.

Since $\gamma \geqslant 1$,

$$
\int_{\Omega \times\{0\}} f(x)|v(x, 0)|^{1-\gamma} d x
$$

is not well defined for all $v \in \mathrm{H}_{0, \mathrm{~L}}^{1}(\mathcal{C})$. In order to study the solvability of problem (1.1), we will analyze the associated approximating problems. Therefore, we introduce a truncated function. For each $A>0$, put

$$
g_{A}(t)= \begin{cases}t^{-\gamma}, & t \in\left[A^{-\frac{1}{\gamma}},+\infty\right), \\ A, & t \in\left[0, A^{-\frac{1}{\gamma}}\right) .\end{cases}
$$

Our first step is to find the weak positive solution of the following problem

$$
\begin{cases}(-\Delta)^{\frac{1}{2}} u=f(x) g_{A}(u), & \text { in } \Omega, \\ u=0, & \text { on } \partial \Omega .\end{cases}
$$

The proof of Theorem 3.2 will be given in the following. Next Lemma 3.3 is from the Proposition 3.1 and Lemma 4.1 in [3]. It will be used in the construction of the existence of weak solution to corresponding approximate problems (3.11).

Lemma 3.3 ([3, 19]). Assume that $\mathrm{f} \in \mathrm{C}^{\alpha}(\bar{\Omega})(0<\alpha<1)$ is a nonnegative function and $\mathrm{f} \not \equiv 0$. Then problem

$$
\begin{cases}(-\Delta)^{\frac{1}{2}} \mathrm{~h}=\mathrm{f}(\mathrm{x}), & \text { in } \Omega, \\ \mathrm{h}=0, & \text { on } \partial \Omega\end{cases}
$$

has a weak positive solution $\mathrm{h}=\left.\operatorname{tr}\right|_{v} \in \mathrm{C}^{1, \alpha}(\bar{\Omega}) \cap \mathrm{C}^{2, \alpha}(\Omega), v \in \mathrm{H}_{0, \mathrm{~L}}^{1}(\mathcal{C}), v \in \mathrm{C}^{1, \alpha}(\overline{\mathcal{C}})$ and $\frac{\partial \mathrm{h}}{\partial n}<0$ on $\partial \Omega$ where $\boldsymbol{n}$ is the unit outward normal on $\partial \Omega$. 
Lemma 3.4. $\varphi=\|\mathrm{h}\|_{\mathrm{L}^{\infty}}^{-\frac{\gamma}{1+\gamma}} \mathrm{h}$ and $\psi=[(1+\gamma) \mathrm{h}]^{\frac{1}{1+\gamma}}$ are weak subsolution and weak supersolution of (3.11), respectively and $\varphi \leqslant \psi$ in $\Omega$ when the parameter $A \geqslant\|h\|_{L^{\infty}}^{-\frac{\gamma}{1+\gamma}}$.

Proof. Proving the conclusion of $\varphi$ is equivalently to prove $(-\Delta)^{\frac{1}{2}} \varphi \leqslant f(x) g_{A}(\varphi)$ in $\Omega$. Easy computations show that

$$
(-\Delta)^{\frac{1}{2}} \varphi=\|h\|_{L^{\infty}}^{-\frac{\gamma}{1+\gamma}}(-\Delta)^{\frac{1}{2}} h=\|h\|_{L^{\infty}}^{-\frac{\gamma}{1+\gamma}} f(x) .
$$

So we only need to prove $\|\mathrm{h}\|_{\mathrm{L}^{\infty}}^{-\frac{\gamma}{1+\gamma}} \leqslant \mathrm{g}_{\mathrm{A}}(\varphi)$.

Case 1. For $x \in \Omega_{1}=\left\{x \in \Omega \mid \varphi(x) \geqslant A^{-\frac{1}{\gamma}}\right\}$, through the directly compute one has

$$
g_{A}(\varphi)=\varphi^{-\gamma}=\|h\|_{L^{\infty}}^{\frac{\gamma^{2}}{1+\gamma}} h^{-\gamma} \geqslant\|h\|_{L^{\infty}}^{\frac{\gamma^{2}}{1+\gamma}}\|h\|_{L^{\infty}}^{-\gamma}=\|h\|_{L^{\infty}}^{-\frac{\gamma}{1+\gamma}}, \text { in } \Omega_{1} .
$$

Case 2. For $x \in \Omega_{2}=\left\{x \in \Omega \mid \varphi(x)<A^{-\frac{1}{\gamma}}\right\}$, by the assumption of Lemma 3.3, we infer that

$$
g_{A}(\varphi)=A \geqslant\|h\|_{L^{\infty}}^{-\frac{\gamma}{1+\gamma}}, \text { in } \Omega_{2} .
$$

Combining Cases 1 and 2, we have

$$
(-\Delta)^{\frac{1}{2}} \varphi \leqslant f(x) g_{A}(\varphi), \text { in } \Omega .
$$

Thus $\varphi$ is a weak subsolution of equation (3.11).

For $\psi$, direct computations show that

$$
(-\Delta)^{\frac{1}{2}} \psi=(1+\gamma)^{\frac{1}{1+\gamma}}(-\Delta)^{\frac{1}{2}} h^{\frac{1}{1+\gamma}} .
$$

On the other hand, since $h$ is a weak positive solution of (3.12), which means $h^{\frac{1}{1+\gamma}}$ satisfies in a weak sense that

$$
(-\Delta)^{\frac{1}{2}} h^{\frac{1}{1+\gamma}}=\frac{1}{1+\gamma} h^{\frac{-\gamma}{1+\gamma}} f(x)
$$

Then we obtained

$$
(-\Delta)^{\frac{1}{2}} \psi=(1+\gamma)^{\frac{-\gamma}{1+\gamma}} h^{\frac{-\gamma}{1+\gamma}} f(x)=[(1+\gamma) h]^{\frac{-\gamma}{1+\gamma}} f(x) .
$$

Therefore it is enough to prove $[(1+\gamma) h]^{\frac{-\gamma}{1+\gamma}} \geqslant g_{A}(\varphi)$ in $\Omega$. Following the same arguments as in proving the subsolution $\varphi$, two cases arise.

Case 1. For $x \in \Omega_{3}=\left\{x \in \Omega \mid \psi \geqslant A^{-\frac{1}{\gamma}}\right\}$, we deduce that

$$
g_{A}(\psi)=\psi^{-\gamma}=[(1+\gamma) h]^{\frac{-\gamma}{1+\gamma}}, \text { in } \Omega_{3} .
$$

Case 2. For $x \in \Omega_{4}=\left\{x \in \Omega \mid \psi<A^{-\frac{1}{\gamma}}\right\}$, it implies $A<\psi^{-\gamma}=[(1+\gamma) h]^{\frac{-\gamma}{1+\gamma}}$. Consequently

$$
g_{A}(\psi)=A<[(1+\gamma) h]^{\frac{-\gamma}{1+\gamma}}, \text { in } \Omega_{4} .
$$

Clearly, Cases 1 and 2 yield that $\psi$ is a weak supersolution of equation (3.11).

Now we are concentrating on proving the inequality $\varphi \leqslant \psi$ in $\Omega$. However, it is trivially. In fact,

$$
\varphi=\|h\|_{L^{\infty}}^{-\frac{\gamma}{1+\gamma}} h \leqslant h^{-\frac{\gamma}{1+\gamma}} h=h^{\frac{1}{1+\gamma}} \leqslant[(1+\gamma) h]^{\frac{-\gamma}{1+\gamma}}=\psi .
$$

This completes the proof of Lemma 3.4.

Furthermore, since $g_{A}(t)$ is nonincreasing in $t$, using the sub-and supersolutions method, we deduce the following Lemma. 
Lemma 3.5. The problem (3.11) has a unique weak positive solution $\mathrm{u}_{\mathrm{A}} \in \mathrm{C}^{1, \alpha}(\bar{\Omega}) \cap \mathrm{C}^{2, \alpha}(\Omega)$ for any fixed $A \geqslant\|h\|_{L^{\infty}}^{-\frac{\gamma}{1+\gamma}}$.

Therefore, in what follows, we will concentrate to prove existence of weak solution to problem (1.1).

Proof of Theorem 2.2. Recalling the definition of $g_{A}(t)$, we clearly see that for fixed t one has $g_{A}(t) \leqslant g_{A^{\prime}}(t)$ while $A \leqslant A^{\prime}$, i.e., $g_{A}$ is no decreasing with respect to subscript $A$. Thus, letting $A=n, n+1, \ldots$ in Lemma 3.4 , by the Maximum principles we obtain

$$
\varphi \leqslant u_{n} \leqslant u_{n+1} \leqslant \cdots \leqslant \psi, \text { in } \Omega,
$$

where $u_{n} \in C^{1, \alpha}(\bar{\Omega}) \cap C^{2, \alpha}(\Omega)$ is the unique weak positive solution of

$$
\begin{cases}(-\Delta)^{\frac{1}{2}} u=f(x) g_{n}(u), & \text { in } \Omega, \\ u=0, & \text { on } \partial \Omega .\end{cases}
$$

Consequently, using (3.13) again, one has a $u \in \mathrm{C}_{0}(\bar{\Omega})$ satisfying $u_{n} \rightarrow u$ in $\mathrm{C}_{0}(\bar{\Omega})$ topology and $\varphi \leqslant$ $u_{n} \leqslant u \leqslant \psi$ in $\bar{\Omega}$. Next, we will be devoted to prove the $u$ is a unique weak solution of problem (1.1).

Recalling $h \in C^{1, \alpha}(\bar{\Omega}) \cap C^{2, \alpha}(\Omega), h>0$ in $\Omega,\left.\frac{\partial h}{\partial \mathbf{n}}\right|_{\partial \Omega}<0$ together with condition $\eta \in C_{0}^{1}(\bar{\Omega}), \eta \geqslant 0$ in $\Omega$, we infer that

$$
\mathrm{m}=\inf _{\Omega} \frac{\mathrm{h}}{\eta}>0
$$

This implies

$$
f(x) h^{-\gamma} \leqslant m^{-\gamma} f(x) \eta^{-\gamma} \in L^{2}(\Omega) \text { and } f(x) \varphi^{-\gamma}=\|h\|_{L^{\infty}}^{\frac{\gamma^{2}}{1+\gamma}} f(x) h^{-\gamma} \in L^{2}(\Omega) .
$$

On the other hand, it follows from Definition 1.2 that there exist a sequence $\left\{v_{n}\right\} \subseteq \mathrm{H}_{0, \mathrm{~L}}^{1}(\mathcal{C})$ which is the weak positive solution of the following problem

$$
\begin{cases}-\Delta v=0, & \text { in } \mathcal{C}, \\ v=0, & \text { on } \partial_{\mathrm{L}} \mathcal{C}, \\ v>0, & \text { in } \mathcal{C}, \\ \frac{\partial v}{\partial \mathbf{n}}=\mathrm{f}(\mathrm{x}) \mathrm{g}_{\mathrm{n}}(v), & \text { on } \Omega \times\{0\}\end{cases}
$$

such that $u_{n}=\left.\operatorname{tr}\right|_{\Omega} v_{n}$ solves problem (3.14) in a weak sense. Consequently, we obtained

$$
\int_{\mathcal{C}} \frac{\partial v_{\mathrm{n}}}{\partial \mathbf{n}} \phi \mathrm{d} x \mathrm{~d} y=\int_{\Omega \times\{0\}} f(x) g_{\mathfrak{n}}\left(v_{\mathrm{n}}\right) \phi \mathrm{dx}, \quad \forall \phi \in \mathrm{H}_{0, \mathrm{~L}}^{1}(\mathcal{C}) .
$$

In particular, using (3.17) with $\phi=v_{n}$, we infer that

$$
\int_{\mathcal{C}} \frac{\partial v_{n}}{\partial \mathbf{n}} v_{n} d x d y=\int_{\Omega \times\{0\}} f(x) g_{n}\left(v_{n}(x, 0)\right) v_{n}(x, 0) d x
$$

Combining $\varphi \leqslant u_{n}$ and $g_{n}(\cdot)$ is non-increasing in $(0,+\infty)$ we infer that the integral in the right hand side of (3.17) can be estimated, by Fatou's Lemma, in the following way

$$
\begin{aligned}
\int_{\Omega \times\{0\}} f(x) g_{n}\left(v_{n}\right) \phi d x & =\int_{\Omega} f(x) g_{n}\left(v_{n}(x, 0)\right) \phi(x, 0) d x \\
& \leqslant \int_{\Omega} f(x) g_{n}(\varphi) \phi(x, 0) d x
\end{aligned}
$$




$$
\begin{aligned}
& \leqslant \int_{\Omega} f(x) \lim _{n \rightarrow \infty} g_{n}(\varphi) \phi(x, 0) d x \\
& =\int_{\Omega} f(x) \varphi^{-\gamma} \phi(x, 0) d x<\infty, \forall \phi \in H_{0, L}^{1}(\mathcal{C}) .
\end{aligned}
$$

Using the Lebesgue dominated convergence theorem, we obtained from the left hand side of (3.17) that

$$
\begin{aligned}
\lim _{n \rightarrow \infty} \int_{\mathcal{C}} \frac{\partial v_{n}}{\partial \mathbf{n}} \phi(x, 0) d x d y & =\lim _{n \rightarrow \infty} \int_{\Omega \times\{0\}} f(x) g_{n}\left(v_{n}(x, 0)\right) \phi(x, 0) d x \\
& =\int_{\Omega} f(x) u^{-\gamma} \phi(x, 0) d x<\infty, \forall \phi \in H_{0, L}^{1}(\mathcal{C}) .
\end{aligned}
$$

Putting together these relations (3.15)-(3.19) we deduce that

$$
\begin{aligned}
\left\|v_{n}\right\|^{2} & =\int_{\mathcal{C}} \nabla v_{n} \cdot \nabla v_{n} d x d y \\
& =\int_{\mathcal{C}} \frac{\partial v_{n}}{\partial \mathbf{n}} v_{n} d x d y \\
& =\int_{\Omega} f(x) g_{n}\left(v_{n}(x, 0)\right) v_{n}(x, 0) d x \\
& =\int_{\Omega} f(x) g_{n}\left(u_{n}\right) u_{n} d x \\
& \leqslant \int_{\Omega} f(x) g_{n}(\varphi) \psi d x \\
& \leqslant \int_{\Omega} f(x) \varphi^{-\gamma} \psi d x \\
& =\int_{\Omega}\|h\|_{L^{\infty}}^{\frac{\gamma^{2}}{1+\gamma}} f(x) h^{-\gamma} \psi d x \\
& \leqslant \int_{\Omega}\|h\|_{L^{\infty}}^{\frac{\gamma^{2}}{1+\gamma}} \psi m^{-\gamma} f(x) \eta^{-\gamma} d x \\
& \left.\leqslant m^{-\gamma}\|h\|_{L^{\infty}}^{\frac{\gamma^{2}}{1+\gamma}}\|\psi\|_{L^{\infty}}\left\|\mathrm{f \eta}^{-\gamma}\right\|_{L^{1}(\Omega)} \quad \text { (since } \mathrm{f \eta}^{-\gamma} \in \mathrm{L}^{2}(\Omega)\right) .
\end{aligned}
$$

Thus the sequence $\left\{v_{n}\right\} \subseteq \mathrm{H}_{0, \mathrm{~L}}^{1}(\mathcal{C})$ is bounded, and hence, up to subsequences (which is still denoted by $\left.\left\{u_{n}\right\}\right)$, converges to $v$ weakly in $\mathrm{H}_{0, \mathrm{~L}}^{1}(\mathcal{C})$ and $\left.\operatorname{tr}\right|_{\Omega} v=u$. So one has

$$
\int_{\mathcal{C}} \nabla v_{\mathrm{n}} \cdot \nabla \phi \mathrm{d} x \mathrm{~d} y \longrightarrow \int_{\mathcal{C}} \nabla v \cdot \nabla \phi \mathrm{d} x \mathrm{~d} y, \forall \phi \in \mathrm{H}_{0, \mathrm{~L}}^{1}(\mathcal{C}) .
$$

Combining (3.17), (3.19), and (3.20), we derive that

$$
\int_{\mathcal{C}} \frac{\partial v}{\partial \mathbf{n}} \phi \mathrm{d} x \mathrm{~d} y=\int_{\mathcal{C}} \nabla v \cdot \nabla \phi \mathrm{d} x \mathrm{~d} y=\int_{\Omega} \mathrm{f}(\mathrm{x}) \mathrm{u}^{-\gamma} \phi \mathrm{d} x, \forall \phi \in \mathrm{H}_{0, \mathrm{~L}}^{1}(\mathcal{C}),
$$

i.e., $v \in H_{0, L}^{1}(\mathcal{C}) \cap \mathrm{L}^{\infty}(\mathcal{C})$ is a weak positive solution of problem (1.2) and $u=\left.\operatorname{tr}\right|_{\Omega} v \in \mathrm{L}^{\infty}(\Omega)$ is a a weak positive solution of problem (1.1).

As the similar arguments in proving of uniqueness in Theorem 2.1, we obtain $u$ is the unique weak positive solution of (1.1). This completes the proof of Theorem 2.2.

\section{Acknowledgment}

This work is supported by the National Natural Science Foundation of China(No.11701443) and the Fundamental Research Funds for the Central Universities (No.300102128108). This work is also supported by the Project Supported by Natural Science Basic Research Plan in Shaanxi Province of China (No. 2017JQ1011, No. 2018JQ1023). 


\section{References}

[1] D. Applebaum, Léry processes-from probability to finance and quantum groups, Notices Amer. Math. Soc., 51 (2004), 1336-1347. 1

[2] B. Barrios, I. De Bonis, M. Medina, I. Peral, Semilinear problems for the fractional Laplacian with a singular nonlinearity, Open Math., 13 (2015), 390-407. 1

[3] X. Cabré, J. G. Tan, Positive solutions for nonlinear problems involving the square root of the Laplacian, Adv. Math, 224 (2010), 2052-2093. 3.1, 3, 3.3

[4] L. Caffarelli, L. Silvestre, An extension problem related to the fractional Laplacian, Comm. Partial Differential Equations, 32 (2007), 1245-1260. 1

[5] M. G. Crandall, P. H. Rabinowitz, L. Tatar, On a Dirichlet problem with a singular nonlinearity, Comm. Partial Differential Equations, 2 (1997), 193-222. 1

[6] Y. Q. Fang, Existence, Uniqueness of positive solution to a fractional Laplacians with singular nonlinearity, Analysis of PDEs, 2014 (2014), 11 pages. 1

[7] A. Garroni, S. Müller, Г-limit of a phase-field model of dislocations, SIAM J. Math. Anal., 36 (2005), 1943-1964. 1

[8] N. Hirano, C. Saccon, N. Shioji, Existence of multiple positive solutions for singular elliptic problems with concave and convex nonlinearities, Adv. Differential Equations, 9 (2004), 197-220. 1

[9] J. L. Lions, E. Magenes, Problèmes aux limites non homogènes et applications, Travaux et Recherches Mathématiques, Paris, (1968). 1

[10] G. Molica Bisci, V. D. Radulescu, R. Servadei, Variational methods for nonlocal fractional problems, Cambridge University Press, Cambridge, (2016). 1

[11] T. Mukherjee, K. Sreenadh, Critical growth fractional elliptic problem with singular nonlinearities, Electron. J. Diff. Equ., 2016 (2016), 23 pages. 1

[12] M. Struwe, Variational Methods, Springer-Verlag, Berlin, (1990). 1, 3

[13] C. A. Stuart, Existence and approximation of solutions of non-linear elliptic equations, Math. Z., 147 (1976), 53-63. 1

[14] Y. J. Sun, S. J. Li, A nonlinear elliptic equation with critical-exponent: estimates for extremal values, Nonlinear Anal., 69 (2008), 1856-1869. 1

[15] Y. J. Sun, S. P. Wu, An exact estimate result for a class of singular equations with critical exponents, J. Funct. Anal., 260 (2011), 1257-1284. 1

[16] Y. J. Sun, S. P. Wu, Y. M. Long, Combined effects of singular and superlinear nonlinearities in some singular boundary value problems, J. Differential Equations, 176 (2001), 511-531. 1, 3

[17] X. Wang, P. H. Zhao, L. Zhang, The existence and multiplicity of classical positive solutions for a singular nonlinear elliptic problem with any growth exponents, Nonlinear Anal., 101 (2014), 37-46. 1

[18] X. Wang, L. Zhao, P. H. Zhao, Combined effects of singular and critical nonlinearities in elliptic problems, Nonlinear Anal., 87 (2013), 1-10. 1, 3

[19] A. L. Xia, J. F. Yang, Regularity of nonlinear equations for fractional Laplacian, Proc. Amer. Math. Soc., 141 (2013), 2665-2672. 3.3

[20] Z. T. Zhang, Critical points and positive solutions of singular elliptic boundary value problems, J. Math. Anal. Appl., 302 (2005), 476-483. 1 